\title{
Theoretical Insight in Financial Decision and Brain as Fractal Computer Architecture
}

http://doi.org/10.21272/fmir.3(2). 91-101.2019

\section{Ana Njegovanović}

Master of Economics, Lecturer at Faculty of Biotechnology in Zagreb; Faculty of Economics and Tourism, University of J. Dobrila in Pula, Croatia

\begin{abstract}
The purpose of this paper is to examine the theoretical interaction of brain dynamics using fractal information tools, fractal geometry in fnancial decision making. This paper concludes a scientific analytical observatory focusing on financial decision making. Through the integration of neuroscientific approach to the brain as a fractal and financial decision-making with the concept of fractal financial market, we open the analytical framework through two interrelated approaches that can increase information on making financial decisions and improve effective financial decisions in times of uncertainty. In order to achieve the aim of the work, the concept of "organized business forms" and the analogue of neurons in the financial market is the fractal - the price of a financial asset, to summarize theoretical basics, multifraktal and financial market / fractal trading, fractal computing architecture. Time series of property prices are dental lines, Fractal Computing Architecture.

Financial decision making is a complex system whose analysis requires a holistic approach including scientific branches (from economics, neuroscience, psychology, mathematics) to understanding the complexity of decision making. Making financial decisions is a complex system whose analysis requires a holistic approach, including scientific branches (from economics, neuroscience, psychology, maths) to understanding the complexity of decision making. Thus, fractal geometry provides a new epistemological framework for the interpretation of real life and the natural world as it is, thus preventing approximation or subjective view. The dynamics of globalization and financial system development opens up new research findings with growing information and financial tools through the use of scientific tools to address complex financial problems.
\end{abstract}

Keywords: fractal geometry, fractal information, financial decision making, brain/neural networks. JEL Classification: G41.

(C) The Authors, 2019. This article is published with open access at Sumy State University.

Cite as: Njegovanović, An. (2019). Theoretical Insight in Financial Decision and Brain as Fractal Computer Architecture. Financial Markets, Institutions and Risks, 3(2), 91-101. http://doi.org/10.21272/fmir.3(2). 91101.2019

\section{Extended introduction}

Fractals consist of self-similarities, and have a fractional Hausdorff-Besicovitch dimension, also called fractal dimension (FD) (Mandelbrot, 1982; Feder, 1988; Falconer 1982; 2003). Fractal geometry was used to study time dynamics, such as the dynamics of the human brain in health (Lutzenberger et al., 1995; Pereda et al., 1998; Eke et al., 2000, 2002; Linkenkaer-Hansen et al., 2001; In addition, B and B et al., 2003, and Gong et al., 2003; Acharya et al., 2005; Bassett et al., 2006, 2010, Hsu et al. (1987), and Zellmann et al., 1997), which give intriguing results (Esteller et al., 1999; Gómez et al., 2009; Zappasodi et al., 2014). For example, it has been shown that FD changes before and during epileptic seizures (Esteller et al., 1999). Fractal analysis and methods of computer intelligence can be used in neuroscience research. Fractal characterization can be used to: estimate invariant scales properties by offering numerical views based on features that complement the usually more complex patterns of neuroscience patterns. Computer intelligent methods can be used to exploit such fractal characterizations, taking into account the possibility of performing analysis of data on nongeometric input spaces, thus overcoming internal boundaries related to Euclidean geometry.

The analysis of complexity of natural objects, and biological systems, show self-similar patterns and scaling properties in the approach of studying the fractal geometry of life. In addition to genomics, proteomics and metabolisms (suffix "omics" refers to the concept of integrity) as a subdiscipline was introduced fractomy (Losa, 2009). Fractal dimensions (Mandelbrot, 1975) introduced fractal analysis in neuroscience - 
Financial Markets, Institutions and Risks, Volume 3, Issue 2, 2019

ISSN (online) - 2521-1242 ISSN (print) - 2521-1250

neurofractolomics. Different types of fractures in life science have been applied to general physiology and pathology (West, 2010) and to clinical neuroscience. The spatial traits of irregular structures that make up the nervous system like brain, cerebellum or cerebral vasculature are interpreted as geometric fractures, and dynamics of the signal of the nervous system can be as dynamic fractures.

The application of fractal geometric approach exponentially increases. Its application goes beyond economics (Mandelbrot, 1977), music (Perez, 2000), medicine (Dela Rosa-Orea, 2014), topology (Vivas, 1999), physics (Aguirre, 2004), astronomy (Martinez, 1999), meteorology (Morales-Acoltzi, 2015), geology (Esper, 2005) and psychology (Prestana, 1999).

Fractal geometry is explicitly included in the science of economics as well as in the areas of organization and business management. The behavior of a company is similar to the behavior of a living being that adapts to its environment (Itturriaga and Jovanovich, 2014). The human factor is the core in making management decisions. Human capital is analyzed by a qualitative and quantitative model (Iwamoto and Takahashi, 2015).

All naturally created by the invariable laws of fractal geometry. This includes static elements as well as energy flows; living beings and their patterns of behavior. They are all built on similar patterns that repeat each other on growing and decaying scales, like Russian nesting pigeons. Different chart levels are not all the same, but they are all self-similar and build one on top of the other based on the basic "code" that is reproduced at different scales. And in a metaphysical and practical sense, the whole universe was built with fractal geometry.

Fractal geometry has expanded our scientific understanding in the field of biology. As the cell functions, it leads to the knowledge and understanding that human beings are biologically. Man is a collection of about 50 trillion cells, each having the same structure and basic functionality, replicated and then adapted to work together to achieve the community's success - the human being. All human body functions are already present in every living cell that contains it. Each station has its own intelligence and all functions of the whole human being. People are programmed by the cells themselves. Fractal geometry has countless economic and financial "behaviors" which, by their very nature, fractal-understanding fractal geometry, enable organizations to improve their profitability and open up completely new economic opportunities that did not exist before. In the 1930s Ralph Elliott suggested that market prices take place in specific patterns, which we now call fractal patterns. Elliot's wave principle manifests itself through appearing fractals over time by expanding and growth as well as collective human psychology. This is reflected in the purchasing and sales decisions that reflect the market price. The principle is popularized by Robert Prechter, a prominent stock market analyst, by understanding that mass psychology manages fractal geometry. In the seventies of the last century, Mandelbrot's research has shown that markets of various kinds show complex behavior that can break into smaller and fewer self-same parts. Price fluctuations can be described as fractal functions. Today, economics and finance are the subject of fractal geometry, used to accurately model risk in the financial market. This understanding has led to the evolution of a new economic discipline called "ecophysics." Another aspect of the real world that fractal finance is is that markets keep "memories" of past moves, especially unstable days, and act in accordance with such a memory. Volatility causes instability; comes in groups and barks according to the laws of fractal geometry.

Brain dynamics are non-linear, and as the most complex natural phenomenon, there are several different and interrelated time scales. In the brain, rapid electrical activity, slow chemical reactions, and even slower diffusion processes were observed. Dynamics is characterized by "no scale" (Stam and de Bruin, 2004; Fraiman and Chialvo, 2012), meaning that certain signal properties are preserved through different time scales. For the description and quantification of such time invariant dynamics, the framework of fractal geometry is often applied (Werner, 2010). Fractals consist of self-similarities, and have a fractional Hausdorff-Besicovitch dimension, also called fractal dimension (FD) (Mandelbrot, 1982; Feder, 1988; Falconer 1982; 2003). Fractal geometry was used to study time dynamics, such as the dynamics of the human brain in health (Lutzenberger et al., 1995; Pereda et al., 1998; Eke et al., 2000, 2002; Linkenkaer-Hansen et al., 2001; In addition, B and B et al., 2003, and Gong et al., 2003; Acharya et al., 2005; Bassett et al., 2006, 2010, Hsu et al. (1987), and Zellmann et al., 1997), which give intriguing results (Esteller et al., 1999; Gómez et al., 2009; Zappasodi et al., 2014). For example, it has been shown that FD changes before and during epileptic seizures (Esteller et al., 1999).

Scaling power and other manifestations of fractal and self-similar patterns in space and / or time can be identified at all levels of neural organization. Progress in the methodology of measuring fractal connections at higher levels of brain organization has led to proliferation of new data. This leads to the request for 
integrating fractality with other insights into the organization and complexity of the brain, especially that the brain is a complex system in the criticality regime, as understood in statistical physics (Chialvo, 2004; Chialvo et al., Kitzbichler et al. ., 2009, Werner, 2007b, 2009a, b). As in other physiological systems that manifest fractal patterns (Bassingthwaighte et al., 1994; West and Deering, 1995; Iannacone and Khokha, 1995; West, 2006), the ubiquity of power-law scaling needs to be addressed in relation to other features of brain organization. We can ask whether there is a link between the fractal organization and the inclination to phase phases of critical systems, and can fractal properties be seen as playing a role for functional integration between different levels of neuronal organization, as Andersen (2000) suggests in an article titled "From Molecules to Consciousness ". Generalizing from the holistic theory of organization and interaction at the molecular level, Agnati et al. (2004, 2009) look at the central nervous system as a nested network at all levels of the organization, on the image of Russian matryoshka dolls: self-similar structures are built into one another. The authors elaborated in detail this theory with "Fractal (Samsonic) Logic" which acted on a set of identical rules that would regulate the relationship between successive levels of the nested system.

\section{Multifraktal and Financial Market / Fractal Trading}

The methods of analysis and prediction of behavior in the financial market are based on neuroeconomics. Neuroeconomics as an interdisciplinary area that combines neuroscience, economics, psychology, mathematics and other disciplines to create the basis for new knowledge about decision-making mechanisms. The analogue of neurons in the financial market is the fractal- the price of a financial asset. Time series of property prices are dental lines. It is not one-dimensional because it is not a direct line, therefore it is not twodimensional at the same time because it does not fill the plane space, so the size of the financial range is between one and two. According to expectations, the fractal dimension of a random time series that is 1 . Fractal is an attractor (boundary and goal) to move the chaotic system. Namely, in the attractor, as well as in the fractal, as it increases, details are revealed (activates the principle of self-similarity). As much as the attractor size changes, it is always at the same rate. Time series are considered fractal when they show statistical self-similar. Self-similarity can be seen while reading ordinary graphs. For example, it is impossible to differentiate minutes, hours and daily charts from any product because they are similar and monotonous. In a technical analysis, a typical example of fractures is "Elliot Waves" whose construction is based on the principle of self-similarity. An additional idea of fractality refers to non-integral dimensions commonly referred to as a one-dimensional, two-dimensional or three-dimensional whole world. However, there may be a non-dimension, such as 2.58 (located between two and three-dimensional). Mandelbrot called such dimensions to fractals. This idea stems from the belief that three-dimensional measurement of the actual ball or cubicle is inadequate, as soon as the real world can hardly find the perfect sphere or cube. In order to describe complex objects, there should be other measurements. Such a measurement of false fractal forms introduces the notion of fractal dimension. From the standpoint of classical Euclidean geometry, the crumpled sheet of paper will be a three-dimensional sphere. But in reality it is still just a two-dimensional paper sheet even if it is crumpled. It can therefore be assumed that the new object will have a dimension greater than two, but less than three. It hardly corresponds to Euclidean geometry, but can be well described by fractal geometry, which claims that the new object will be located in a fractal dimension of approximately 2.5 (it will have a fractal dimension of about 2.5).

For fractals and multifractals there is an extensive mathematical basis. Fractal patterns appear not only in changes in the price of securities, but in the distribution of space galaxies, in the shape of the coast and in decorative designs generated by countless computer programs.

Fractal is a geometric shape that can be divided into parts, each of which is a reduced size of the whole. In finance, theoretical reformulation of market folklore - namely, movements in stocks or currencies look just as the market price increases or decreases to match the same price and price. The observer can not then determine which data relates to prices that change from week to week, day or hour. This quality defines graphs as fractal curves and makes available many powerful tools of mathematical and computer analysis.

Individual investors and professional stock market traders and currencies know that prices quoted on any financial market often change with acceleration. Distances have been made and lost in the sudden bottlenecks of activity when it seems that the market is accelerating and volatility is increasing. Modern portfolio theory seeks to maximize yields for a certain level of risk. The mathematical core theory of the portfolio addresses extreme situations with benign neglect: she thinks that large market moves are too likely to be important or impossible to take into account. Market reality is different. The risk reduction formula behind the portfolio 
Financial Markets, Institutions and Risks, Volume 3, Issue 2, 2019 ISSN (online) - 2521-1242 ISSN (print) - 2521-1250

theory relies on a number of demanding and unfounded premises. First, price changes are statistically independent of each other (today's price does not affect the change between the current price and tomorrow). As a result, predictions of future market trends become impossible. Another assumption is that all price changes are arranged in a pattern that matches the standard bell curve. The width of the ring pattern (measured by its sigma or standard deviation) shows how far the price changes differ from the mean; Extreme events are considered extremely rare. Do financial information conform to such assumptions? Charts of stock or currency changes over time reveal a constant background of small price and price movements - but not the same as would have been expected if the price changes match the bell curve. However, these forms are just one aspect of the chart. Big price leaks are becoming more common as market turbulence is growing - clusters appear on the chart. Fractal geometry allows the model to reproduce patterns that characterize the peaceful markets of portfolio theory as well as the harsh trading conditions of recent months. The described method of creating a fractal model of prices may change to show how market activity accelerates and slows down - there will be volatility. This variability is the reason that the prefix "multi-" added the word "fractal".

To create a multifractal from a singlefractal extension or shortening the horizontal timing axis so that the parts of the generator are either stretched or squeezed. At the same time, the vertical axis price may remain untouched. The first part of the unifractal generator is gradually shortened, which also provides a space for extending the second part. After these adjustments, the generators are multiplied. Market activity accelerates in time intervals. Such a generator change can produce a complete simulation of the price fluctuation in the given period, using the interpolation process. Each time the first part of the generator is shortened - and the process of sequential interpolation - creates a graph that increasingly resembles the characteristics of the unstable markets and represents the first part of the generator and decelerates to the interval corresponding to the second part. Instead, multifractals can be placed in the "stress test" portfolio function. In this technique, rules based on multifractals are trying to create the same patterns of variability as well as unknown rules that govern the actual markets. Multifractals accurately describe the relationship between generator shapes and up-down pricing patterns found on real market data charts. At a practical level, based on historical market data, a fractal generator can be developed. The model used not only checks what the market did yesterday or last week. This is a more realistic view of market fluctuations called fractional Brown's movements in the time of multifractal trading. Graphs generated by generators produced by this model can simulate alternative scenarios based on previous market activity. These techniques are not approaching predicting a fall in prices or a rise on a given day based on the records that have been made so far. But they provide estimates of the likelihood that the market could make and allow to prepare for inevitable sea change. New modeling techniques have been designed to throw light on the seemingly impenetrable financial market lurch. They also recognize the sailor's warning that, as the recent events show, deserve to be listened to: even in the brilliant sea, the storm can only be above the horizon.

Traders always strive to integrate new techniques into their market analysis to take advantage. The fractal pattern is a repeating pattern in which the same configuration appears in the structure, in different scales. So, in nature, we see fractal patterns in the growth of crystals, branches of tree branches and the structure of flakes, to name but a few. They show the characteristics of recursiveness and self-similarity. Fractal patterns are recursive, because regardless of the complexity of the whole, it is constructed of many repetitions of the same process. They are self-similar, that if you look at a small part of the sample, it does not differ much from much larger part of the sample or even whole. But how to link the relationship between the fractal and the financial market in an analogous way. By observing market price movements as a whole that is extremely complex perhaps and by accident. Fractal theory, suggested by well-known retailer and analyst Bill Williams, suggests that this complexity is built from self-similar patterns of merchant behavior. Ultimately there is an inconsistent structure as a whole that we can understand.

Williams argued that Elliott's wave was the fundamental structure of the market and that Elliott's wave structure is fractal. If trades are the result of fractal behavior, Williams concludes, then aggregate behavior also follows a fractal pattern. In short, recognizing the fractal pattern of behavior of the total mass of retailers offers a way to potentially profit from the market. Bill Williams has claimed that these fractal patterns are simple and easily recognizable. However reality is different. Bill William's fractal indicators are the standard tools that come with the MetaTrader 4 trading platform. Manually, there is no need for a separate pickup of a fractal display (see detailed view: MetaTrader 4-EURUSUD Hourly Chart-Data Range: 17 Apr, 2016 -24 Apr 2017 and Dana Range: 23 Jan. 2017-2 Mar, 2017). Fractal trading is designed to align with the trend. The fractal indicator quickly identifies fractal elevations and declines that may be significant, and we get signals 
that are intended for alignment with the directed market current. In this case, the standard fractal indicator in MT4 is not the only game in the city, and other customized indicators. There are, of course, reversed fracture indicators that focus on recognizing price fluctuations.

There are even fictitious fractal indicators with alert warnings that we can customize, which inform us when key forms are formed. It is rational that we do not rely too much on one indicator in isolation. We can also conclude that fractals are more effective as a way to confirm or support our decision-making, rather than as a primary signal to trigger a trade. There are a number of available combinations. Which is the best way to decide - a good way to discover effective combinations is to experiment with the risk free environment offered by a demo trading account. Once we see what suits us, we can make a transition to live markets and see if the strategies will lead to success. However, it is important to note before deciding any investment decisions to seek experts to ensure understanding of the risk.

\section{Theoretical basics}

Fractals have two specific properties: they consist of parts; similarities, and have a fractional HausdorffBesicovitch dimension, also called the fractal dimension (FD) (Mandelbrot, 1982, Feder, 1988, Falconer, 1982, 2003). Fractal geometry was used to study time dynamics, such as the dynamics of the human brain in health (Lutzenberger et al., 1995; Pereda et al., 1998; Eke et al., 2000, 2002; Linkenkaer-Hansen et al., 2001; In addition, B and B et al., 2003, and Gong et al., 2003; Acharya et al., 2005; Bassett et al., 2006, 2010, Hsu et al. (1987), and Zellmann et al., 1997), which give intriguing results (Esteller et al., 1999; Gómez et al., 2009; Zappasodi et al., 2014). For example, it has been shown that FD changes before and during epileptic seizures (Esteller et al., 1999). The fractal approach encompasses a modern mathematical field that describes Euclidean geometry of nature irregularities, which implies fragments that lead to fractals. Auto-similarity and fractal dimensions are major features (Williams, 1997). First indicates that each entity retains the same format as its global characteristics. Fractals represent an incomplete dimension that goes beyond the topology of the figures shown. (Braña, 2003), which provides a better description of natural processes. Two methods can be used to describe these methods; repeating the linear fractal process and applying complex non-linear fractal numbers (Mandelbrot, 1997).

Fractality or self-similar structures in a complex network can be one of the features that can explain the functional relationships of a larger network to the fundamental structure through different levels of organization (Song, C., Havlin, S, and Massey, H. 2005, 2006). The theory of scaling and renormalization can highlight the importance of flow of information in a complex network and its self-organization (Rozenfeld, H. D., Song C., and Makse, H. A., 2010). It has been shown that the hierarchical organization of modules in functional brain networks (fMRI) shows fractal properties (Gallos, L. K., Makse, H. A. \& Sigman, M. A). This fractal organization of the module in the network holds the nodes firmly bound within the corresponding modules and uses nodes of low degree as inter-modular connectors showing disasortative topology (Barabási, A.L. and Albert, R., 1999). Furthermore, it has been shown that networks (forty-seven different networks) that are structurally self-organized follow the fractal law in the area of network size and edge density (Laurientia, PJ, Joyceb, KE, Telesfordb, QK, Burdette, JH \& Hayasaka, 2011). , the question remains whether the organization of modules and modules at different topological levels will follow the fractal nature or not and whether the fractal properties of the brain networks of different organisms (lower to higher levels) are not fully investigated.

If we look at a human brain that has an estimated $3.5 \times 10^{15}$ bytes memory at a rate of 2.2 petaflops (1015 operations per second), it is about 1.21 and consumes 20 watts in power. For comparison, the fastest supercomputer in the world in June 2011 was Fujitsu's K computer with $30 \times 10^{15}$ bytes of memory, running at 8.2 petaflop speeds, small storage size, and 12.6 megawatts. In June 2012, K's computer was downgraded to the world's fastest computer by US-based IBM Sequoia, the supercomputer Blue Gene / Q that works on 16,325 petaflops using $123 \%$ more processor processors and consumes only $7.9 \mathrm{MW}, 37 \%$ less than the. The race is for the fastest and most powerful supercomputer in the world. The large amount of energy consumed by current computers generates a large amount of heat that requires cooling systems that consume even more energy. The human brain is far better in terms of size and energy consumption.

The growing complexity of financial problems and the huge amount of financial data often make it difficult to apply traditional models and algorithmic procedures. Well, the field of computer intelligence gives arsenal of useful techniques. These techniques include new risk-and-uncertainty modeling tools, data mining 
Financial Markets, Institutions and Risks, Volume 3, Issue 2, 2019

ISSN (online) - 2521-1242 ISSN (print) - 2521-1250

techniques for complex database analysis, and powerful algorithms for complex optimization issues. Computer intelligence has also been rapidly evolving over the last few years and is now one of the most active areas in operations and computer science research.

Fractal models provide an alternative story of the nature of the market. Early developmental versions of the fractal formulation of the market were proposed at the beginning of the 20th century. In the thirties of the last century, Elliot (Frost and Prechter, 1998) disputes the assumption that the market has no memory indicating that certain patterns (waves) tend to appear in it. Each of Elliot's waves could be broken down into portions that resembled the original wave, to fractal similarity. The existence of structures represented by a graphical path is not possible by a random walk hypothesis. However, it should be emphasized that no matter how accurate his observation was, Eliot did not construct any statistical or mathematical theory that could strictly support his point of view. On the other hand, in the 1960s, Mandelbrot (Mandelbrot and Hudson, 2004) proved that some property did not respect the Gaussian statistics imposed by the random walk hypothesis. Mandelbrot has shown that property prices instead show statistically self-similar behavior. With more precision, Mandelbrot argued that financial time sequences can be modeled as partial Brown moves (fBm), a series whose roughness can be characterized by a constant called Hurst exponent $(\mathrm{H})$. For the fBm series, Hurst's exponent values range between 0 and 1 . Free to say, as the time series $\mathrm{H}$ approaches 0 , the series seems to be a bit weird, and as $\mathrm{H}$ approaches 1 , it seems to be more correct. Hurst's exponent is related to the dimension of the fractal and can be shown to be also the measure of the memory of the series: for $\mathrm{H}>0.5$, the increment series are positive by autocorrelation, while for $\mathrm{H}<0.5$ are negatively autocorrelated. Case $\mathrm{H}=0.5$ and is suitable for random walk. Hurst's exponents of real property have been shown to vary between 0.35 and 0.65 and not exactly 0.5 (Sang, Ma and Wang, 2001). Therefore, Mandelbrot's theory can be considered as a generalization of the random walk hypothesis. Mandelbrot's model was much more complicated than random walk theory and could not deal with mathematical behavior. Mandelbrot and Hudson wrote in 2004 (Mandelbrot and Hudson, 2004) that fractal theory was not a tool for prediction.

However, over the last few years, and especially after the last series of global financial crises, new interest in the model has emerged. Investors and traders have realized that tools they use may not be suitable for describing extreme phenomena, such as bubble formation and acute price falls that are more frequent than expected classical theory. This new interest manifested itself through research to determine if the market is fractal or not ( Parthairathy, 2013; Malavoglia, Gaio, Júnior and Lima, 2012; Ling-Yun; 2011; Onali and Goddard, 2011; Sun; Rachev and Fabozzi; 2007; In and Kim, 2006). An attempt at prediction and market based on its fractal properties (Duchon, Robert and Vargas, 2012; Richards, 2004; Cui and Yang, 2009). New theories and investment strategies, combined with fractal models with previous formulas, the Black-Scholes formula, were developed (Bayraktar and Poor, 2005). Fractal analysis also has applications in macroeconomics (Blackledge, 2008). Recently, innovative developed approaches are multifractals (Dezsi and Scarlat, 2012; Schmitt, Ma and Angounou, 2011).

Neuron is a type of cell that processes the information it receives from other neurons. Synapse is the connection between the neurons that allow the flow of information from one neuron to another. However, in neural networks, the node acts as a neuron receiving information, performing a quantitative function that transforms information. The synapse transfers its data from one node to another, and also adjusts the data. When the node sends a signal that synapses a lot of information by multiplying data with a constant value (weighting) and although it can accumulate data from multiple synapse connections and add all that information before processing just like the synapses that work and customize data by multiplying by value (nodal bias). Nodes are activated when its output is large enough to pass a certain threshold defined by the computer programmer. It is a very simple model although most artificial nerve networks contain thousands of neurons.

The Artificial Neural Network is a computational architecture based on the design of mutual neuron bonds in our brain. There are many variations, but one of the simplest is the feed-forward network. Neural networks have many practical applications. Each unit makes a simple calculation as a supplement or application of a simple function. It takes data from many other neurons and aggregates those data that comes and sends it downstream to other neurons. These things are connected to a kind of network. Every computer works through algorithms. Algorithms are mathematical recipes that provide instructions to complete a particular task. The algorithms provide a very specific set of instructions that leave nothing to the interpretation. What the machine learning differs from this old frame is that in this case algorithms can change their own instructions to improve the function of the computer and that is actually the core of artificial intelligence. While ordinary machines 
feature speed and accuracy, they are limited by program parameters. Human intelligence is, on the other hand, much more adaptable to actually achieve artificial intelligence, computers have to think flexibly and there are many approaches to making computers more competitive than human brain, but some computer programmers have concluded this well if they can not win them to join them. One popular technique for creating artificial intelligence is actually designed to work as a human brain and nervous system, called an artificial neural network. The specific fractal characteristics of the networks allow to perform a new type of data processing. Their macro-architecture is defined by fractal algorithms. Microstructures can use the principles of conventional neural networks to modulate the response of each neural column to the history of network learning. Each signal will pass many small neural networks (in the form of neural columns) on the road through the entire network, which therefore acts as a network.

The abstract network structure is dialectical with multiple binary trees in which activity is propagated in wavefronts, whose interference allows the whole network to participate in data processing. There are significant correspondences with biological neural networks, which may be the consequence. reflect the principles of fractal algorithms in morphogenic events. Further research on these structures promises to provide an opportunity to create a new kind of neural networks and to improve our knowledge of the function and structure of biological nerve systems. Namely, Biological Central Nervous Systems with Massive Parallel Structures and Recursive Projections show fractal features in structural and functional parameters (Babloyantz and Louren, 1994). Juliet sets up and Mandelbrot's set are known classic fractals with all their compositional, deterministic chaos and beauty, generated by repetition of nonlinear functions. Appropriate algorithms can be transposed, based on their geometric interpretation, directly into the massive parallel structure of neural networks that work on recursive projections.

The implementation of fractal learning can improve the work of artificial neural network. Fractal refers to a mathematical set that can display a repeating pattern that is displayed on each scale. It is also known as evolving symmetry or growing symmetry. If replication pattern is the same on each scale it is called a selfsimilar pattern. In order to link all AI research, so that research in one field can benefit from achievement in the second, a new kind of framework emerged. This framework emphasizes the common elements of all learning tasks, so that general algorithms can be devised for a wide range of learning tasks, so algorithms can be effectively compared. The fractal framework is called fractal growth or fractal learning. Fractal learning in artificial neural networks facilitates the deployment of dynamic automation in artificial neurons.

\section{Fractal computing architecture}

The research carried out by Subrata Ghosh, Krishna Aswani, Surabhi Singh, Satyajit Sahu, Daisuke Fujita and Anirban Bandyopadhyay on computer-like brain construction, 2014, shows that a new computer paradigm of Gödel's concept of engine incompletion is achieved by a combination of classical, semi-classical and quantum mechanics together creating multi-layered worlds with different clocks. The decision-making protocol is not a logical reduction in the decision of projection of frequent fractal operations in real space, this is the engineering perspective of Gödel's theorems of incompleteness. This research would bring human brain robots (Bandyopadhyay, A .; Miki, K. A., 2013). Or creative and intelligent followers for industrial use and opening a new physical world of biology, parallel to chemical genetic and molecular biology today.

Hippocampus (Tsuda, I., Yamaguti, Y .; Kuroda, S .; Fukushima, Y .; Tsukada, M., 2008), the most prominent organ is a hippocampus. The basic architecture is a helix molecular assembly with a large number of cables radiating from it radially on different helical lengths. In the artificial brain as proposed by the authors (Subrata Ghosh, Krishna Aswani, Surabhi Singh, Satyajit Sahu, Daisuke Fujita and Anirban Bandyopadhyay, 2014) each individual loop can receive signals with quantized phase differences. Self-regulating neurons in the network to generate a unique spring in the hippocampus region (all four layers) with the notion that prefrontal cortex, thalamus, hypothalamus on all seven (or eight, avoiding hardware debate) sub-organs of the middle brain are associated with neuronal fibers.

The functional model of Amigdale (Prévost, C., McNamee, D .; Jessup, R.K .; Bossaerts, P. O'Doherty, J.P. Evidence, 2013) is a filter that helps the human brain in selecting one of the many possible choices of the frequency fracture of the seed. During making decisions, the human brain gets the final fused frequency fracture in the hippocampus, which contains all possible decisions and solutions. Amigdale's hardware looks like a seed with a large number of fractal aids surrounding a complex neuron circuit, changing its axiom by 
Financial Markets, Institutions and Risks, Volume 3, Issue 2, 2019 ISSN (online) - 2521-1242 ISSN (print) - 2521-1250

constructing a distinctive fractal species with the remains saved in Amigdala by birth and evolution with our life practices.

Thalamus is the entry into the sensors, the entrance to the brain and the entrance to the cerebellum (Tian, X., Xiao, Z.G., 20005). It's a universal synchronizer. The machine looks like a few springs with them, if one of them is activated, then complex vibration is automatically activated across the entire system. Each spring-like structure accepts a certain kind of sensory signal. Nature has this body that is constructed with self-compatible neurons and all input outlets are fed verally across this hardware.

Pre-frontal cortex controls futuristic simulations (Oku, M., Aihara, K. A, 2008). This region is directly connected to the frontal lobe where the highest levels of fractal seed are stored.

The hypothalamus in the normal human brain is used for vital motor control and creates rhythms (Tsuda, I., Yamaguti, Y., Kuroda, S., Fukushima, Y., Tsukada, M. A, 2008). These programmed activities are not essential to the computer that is to be built (Subrata Ghosh, Krishna Aswani, Surabhi Singh, Satyajit Sahu, Daisuke Fujita and Anirban Bandyopadhyay, 2014) but it is supreme authority if the transformation is to the highest level in the final decision-fractal in the hippocampus .. Then the protocols can be programmed here. Neurons are similar to themselves on an LC coupled oscillator type periodic fractal semiconductor generator and neuron antennas to convert the final fractal decision into certain boundaries. This is the point of connecting users to modulating the computer process in the artificial brain.

Cerebelum reads the final decision of the fractal from the Hippocampus and its hardware is a fractal network of trees like neurons (Schmahmann, J. D., Caplan, D., 2006). It sends extremely synchronized signals to all sensors for better data collection to improve the decision making process.

Basal Ganglia conducts process learning or practice in practice (Beiser, DG, Hua, SE, Houk, JC, 1997. Ritch, S. Mathematical Model of Basal Ganglia and Oscillatory Neuron Clusters in Relation to Parkingsonian Tremors. /www.math.duke.edu/mathbio/documents/ reu-posters / rich_poster.pdf (accessed on 15 January 2014). Basal Ganglia changes the ultimate frequency of decision-making, the fractal of the hippocampus so that it is systematically captured and the brain of the brain can specifically reconfigure the neuron circuit. Therefore, the basal ganglia creates a new type of frequent fracture that allows him to control multiple sets of brain-generated solutions at a distance of $200 \mathrm{~ms}$, in this sense this is one of the most important organs in the brain, maintaining continuation among discrete computer input-output processes.

\section{Instead of a conclusion}

Scientific models are defined as abstract representations based on scientific rules, reducing the complexity of the problem situation. Within the business analyst's model, it tries to eliminate those real-world details that do not affect the relevant goals of the problem. The model will reveal what the creator believes in solving the problem. According to Curtis and the rest. (Curtis, 1992), this insight and understanding of the problem will be the basic building elements for the study of the system. Dijkstra (Dijkstra, 1992) discovered the idea of structuring problems through models. In many natural cases the observer could describe as chaotic and accidental. Forms that can be described by some kind of mathematical formula.

Using the concept of "organized forms of business activities", fractals can be introduced. Fractal is defined as a form that can be broken into smaller portions each of which echoes the whole. As data sets become more and more complex, applications based on artificial neural networks become more and more present. Organizations must focus on investing in such skills and staff training so they can build and interpret fractal model building and thus develop effective artificial neural networks.

Fractal applies artificial intelligence to solve complex, real-life problem scales. Fractal has built a unique data entry, instrumentation, and analysis platform that enables encoding relationships between data in the form of graphs through ontology and taxonomy - called Fractal OS. The Fractal OS TM platform provides tools for gathering, organizing and enriching network data, operational data, and financial data across the enterprise and the web to support fact-based decisions and effectively explore potential or hypothetical outcomes.

Mandelbrot (1975) points out "the markets are fractal" describing repetitive or self-similar mathematical patterns. "Fractal is a geometric shape that can be divided into parts, each of which is a reduced size of the whole. In finance, this concept is not an abstraction without roots, but a theoretical reformulation of market folklore - namely, that movements of stocks or currencies look similar when a market map is enlarged or reduced to match the same price and price. The observer can not then determine which data relates to prices 
Financial Markets, Institutions and Risks, Volume 3, Issue 2, 2019

ISSN (online) - 2521-1242 ISSN (print) - 2521-1250

that change from week to week, day or hour. "). While Robert Hagstrom in his book, Investing in the Last Liberal Art, elaborates: "Fractal mathematics can not predict the results that result from complex adaptive systems, but we can tell that such results will inevitably occur. All that can then be done is to prepare for their arrival and wait".

The speed of real changes and the potential for new mass moves have never been bigger. As Charlie Munger says, "The great lesson in microeconomics is to differentiate when technology will help you when it will kill you ... there are all kinds of wonderful new inventions that give you nothing as owners except the ability to spend much more money in the business that will and still be bad. The money will still not come. All the benefits of a major improvement will go to customers. "

"Types of feedback loop that can lead to social, economic and technical changes are placed on steroids in this hyper-linked digital environment. If you think you understand all the implications of this increase in "interdependence", you do not understand the problem". We can refer to Paul Samuelson at the end: "Benoit Mandelbrot was irrefutable the original mind, and economists were blessed with his insights. In the wake of the great non-economists who, with the help of quantum leaps, an advanced economy, along with John von Neumanna, read the name of Benoit Mandelbrot "(Paul Samuelson, Gauss's Self-Affinity).

Christian Koch, a leading researcher on consciousness and the human brain, has famously called the brain "the most complex object in the known universe." We can be fascinated with a hundred billion neurons and a hundred trillion connections, the brain is a dizzyingly complex object ,.But there are plenty of other complicated objects in the universe (galaxies can group into enormous structures - called clusters, superclusters, and filaments- that stretch for hundreds of millions of light-years ). The human brain's computing is significantly slower, but more flexible and dynamic in that neurons handle complex problems in a massively parallel way and during this processing, new neuron connections are formed to optimize the solving performance. The net result is an unparalleled capacity to solve highly complex tasks simultaneously, such as coming up with string theory. Another major drawback of digital computers compared with biological ones is that these are only as good as their programming. The software that took Apollo 11 to the Moon had approximately 145,000 lines of code, the Mars rover Curiosity 2,5 million, and Mac OS X 86 million ( Wolbring, G., Diep, L., Yumakulov, S., et al., ) . So imagine if it would be possible to create computers that work out complex problems in similar ways as biological brains do, by self-assembling information and writing its own program to solve a particular problem.

Subrata Ghosh (his Ph.D. in organic synthesis from the Indian Association for the Cultivation of Science, India, in 2010, working on the total synthesis of biomolecules ) and Anirban Bandyopadhyay (Senior Scientist at the National Institute for Materials Science (NIMS), Japan, and currently a visiting professor at MIT, USA) and co-workers describe ( Wolf, J.G. 2013 ): the fundamental computing principle of a frequency fractal brainlike computer that does not need circuits or programming . Organic "brain jelly" computer consists of proteinlike particles that start to self-assemble, triggered by an electromagnetic signal . Essentially, this multilayered hierarchical self-assembly functions in the same way that proteins are assembled from individual amino acids to a secondary and finally to their three-dimensional and active form. In the brain jelly, self-assembly leads to the formation of a basic artificial neuron structure, with various three-dimensional nano-structured assemblies, i.e., wire, spiral, spherical, or semicircular assemblies.

"Brain jelly is a chemical soup made in such a way that for any argument it learns, an equivalent nano-structure is formed that holds the argument. It learns arguments like, "if there is a rain, then take out umbrella". The vibration for "if-rain", say $15 \mathrm{~Hz}$, continues for more than a certain time in a nanowire, then the "then-takeout-umbrella" vibration of $30 \mathrm{~Hz}$ will take over in that nanowire. This we call transformation from "if" to "then" or decision making. The more arguments the jelly learns, the more materials self-assemble as connected nanowires. Eventually we get a network of nanowires. If we neglect the material, in abstract terms, it is a collection of vibrations in a giant 3D network. This jelly network is our computer. Any small 3D pattern of vibrations, if pumped into this network, first expands and all associated patterns start vibrating. Subsequently, transformation into the most associated pattern occurs, which is radiated out and captured outside the brain jelly as the final result of the input. Look at it this way: the input pattern contains our thoughts and the output 3D pattern is our decision. This is how brain jelly computes", suggests research by Dr. Bandyopadhyay.

Most of the fractal physical nature of brain analysis is focused on the gross or physical nature of the brain by techniques such as MR (Sandu et al., 2007) or the study of the shape of certain neurons (Milosevic, et al., 2007). But both approaches ignore the subcellular neuronal structure which contributes to the physical fractal 
Financial Markets, Institutions and Risks, Volume 3, Issue 2, 2019

ISSN (online) - 2521-1242 ISSN (print) - 2521-1250

nature of these structures. Concept tensegrity could be useful in understanding the physical structure of the brain (Gardiner et al., 2008). Tensegrity architectures are based on the self-sustainability of tensile and compression resistance components. In the cell, it implied that actin microfilaments act as compressionresistant compression components (Wang et al., 1993). It should be noted that each of these structures consists of amino acids that can overlap according to tensegrity principles (Zanotti and Guerra, 2003). One of the theses is that the tensegrity theory is that the building block of any tensegrity system triangles. The triangle is a basic building block. This indicates a structure in the brain similar to a Sierpinski triangle that leads to a triangular fractal with smaller triangles.

Financial decision making is a complex system whose analysis requires a holistic approach including scientific branches (from economics, neuroscience, psychology, mathematics) to understanding the complexity of decision making. Thus the fractal geometry of Nature Benoit Mandelbrot has provided a new epistemological framework for the interpretation of real life and the natural world as they are, preventing approximation or subjective view.

The dynamics of the development of globalization and financial systems opens new insights into research.

\section{References}

1. Agnati L. F., Baluska F., Barlow P. W., GGuidolin D. ( 2009). Mosaic. Self- similarity logic, and biological attraction. Commun. Integr. Biol. 2, 552-563.

2. Agnati L. F., Santarossa L., Genedani S., Canela E. I., Leo G., Franco R., Woofs A., Lluis C., Ferre S., Fuxe K. (2004). On the nested hierarchical organization of CNS: basic characteristics of neuronal molecular organization in Cortical Dynamics LNCS 3146 (ed. Erdi P., editor. ), 24-54, Springer, Berlin

3. Albert R., Barabasi A. (2002). Statistical mechanics of complex networks. Rev. Mod. Phys. 74, 479710.1103/RevModPhys.74.47

4. Andersen C. M. (2000). From molecules to mind: how vertically convergent fractal time fluctuations unify cognition and emotion. Conscious. Emot. 1, 193-22610.1075/ce.1.2.02and

5. Babloyantz A, Louren, C (1994) Computation with Chaos: A paradigm for cortical activity, Proc Natal Acad Sci USA, 91, 9027-9031.

6. Bandyopadhyay, A.; Miki, K. (2013). A vertical parallel processor. JP-5187804.

7. Baraasi, A,-L and Albert, R. (1999). Emergence of scaling random networks. Science 286, 5019-512.

8. Beiser, D.G.; Hua, S.E.; Houk, J.C. (1997). Network models of the basal ganglia. Curr. Opin. Neurobiol., 7, 185-190.

9. Dijkstra, E. (1979). Programming Considered as a Human Activity. Classics in Software Engineering, Yourdon Press: New York.

10. Dorogovtsev S. N., Mendes J. F. F. (2000). Evolution of networks with aging of sites. Phys. Rev. E62, 1842-184510.1103/PhysRevE.62.1842

11. Dorogovtsev S. N., Mendes J. F. F. (2001). Evolution of networks. Adv. Phys. 51, 1079 $118710.1080 / 00018730110112519$

12. Dorogovtsev S., Mendes J. (2003). Evolution of Networks: From Biological Nets to the Internet and WWW. New York: Cambridge University Press.

13. Fraiman, D., and Chialvo, D.R. ( 2012). What kind of noise is brain noise: anomalus scaling behavior oft he resting brain activity fluctuations. Front.Physiol. 3:307.

14. Gallos, L.K., Makse, H.A. and Sigman, M. ( 2012 ). A small world of weak ties provides optimal global integration of self-similar module sin functional brain networks. Proc. Natl. Acad. Sci. USA 109, $2825-$ 2830

15. Gardiner J, Marc J, Overall R. (2008). Cytoskeletal thermal ratchets and cytoskeletal tensegrity: determinants of brain asymmetry and symmetry? Front Biosci; 13: 4649-4656.

16. Hilgetag C. C., Koetter R., Stephan K. E., Sporns O. (2002). Computational methods for the analysis of brain connectivity, in Computational Anatomy, ed. Ascoli G. A., editor. (Totowa, NJ: Humana Press; ), $295-335$.

17. Hilgetag C. C., Koetter R., Stephan K. E., Sporns O. (2002). Computational methods for the analysis of brain connectivity, in Computational Anatomy, ed. Ascoli G. A., editor. (Totowa, NJ: Humana Press; ), $295-335$.

18. Laurientia, P. J., Joyceb, K. E. (2011). Telesfordb, Q. K., Burdettea, J.H. and Hayasaka. S. Universal fractal scaling of self-organized networks. Physical A 390, 3608-3613 
19. Losa GA. (2009). The fractal geometry of life. Riv Biol 102(1):29-59

20. Mandelbrot BB. (1975). Stochastic models for the earth's relief, the shape and the fractal dimension of the coastlines, and the number-area rule for islands. Proc Natl Acad Sci U S A 72(10):3825-8.

21. Mandelbrot BB. (1983). The fractal geometry of nature. New York: W.H. Freeman \& Co.

22. Mandelbrot B. B., van Ness J. W. (1968). Fractional Brownian motions, fractional noises and applications. SIAM Rev. 10, 422-43710.1137/1010093

23. Milosević NT, Ristanović D, Gudović R, Rajković K, Marić D. (2007). Application of fractal analysis to neuronal dendritic arborisation patterns of the monkey dentate nucleus. Neuroscience Letters; 425: 23-27.

24. Oku, M.; Aihara, K. (2008). A mathematical model of planning in the prefrontal cortex. Artif. Life Robot., $12,227-231$.

25. Park J. P., Newman M. E. J. (2004). Statistical mechanics of networks. Phys. Rev. E, 70, 066117.10.1103/PhysRevE.70.066117.

26. Prévost, C.; McNamee, D.; Jessup, R.K.; Bossaerts, P.; O’Doherty, J.P. (2013). Evidence for model-based computations in the human amygdala during pavlovian conditioning. PLoS Comput. Biol., 9, e1002918.

27. Roerig B., Chen B. (2002). Relationships of local inhibitory and excitatory circuits to orientation preference maps in Ferret visual cortex. Cereb. Cortex 12, 187-19810.1093/cercor/12.2.187

28. Rozenfeld, H. D.., Song, C. and Makse, H. A. (2010). Small-world to fractal transition in complex networks: a renormalization group approach. Phys. Rev. Lett. 104, 025701.

29. Schmahmann, J. D., Caplan, D. Cognition, emotion and cerebellum. Brain 2006, 129, 290-292.

30. Sandu AL, Rasmussen IA Jr., Lundervold A, Kreuder F, Neckelmann G, Hugdahl K, Specht K. (2007). Fractal dimension analysis of MR images reveals grey matter structure irregularities in schizophrenia. Comput Med Imaging Graph, 32, 150-158.

31. Sporns O., Koetter R. (2004). Motifs in brain networks. PLoS Biol. 2, e369.10.1371/journal.pbio.0020369

32. Stam C. J., de Bruin E. A. (2004). Scale-free dynamics of global functional connectivity in the human brain. Hum. Brain Mapp. 22, 97-10910.1002/hbm.20016

33. Stam C. J., Reijneveld J. C. (2007). Graph theoretical analysis of complex network in the brain. Nonlinear Biomed. Phys, 1, 3.10.1186/1753-4631-1-3

34. Stam C. J. (2005). Nonlinear dynamical analysis of EE and MEG: review of an emerging field. Clin. Neurophys. 116, 2266-230110.1016/j.clinph.2005.06.011

35. Song, C., Havlin, S. and Makse, H.A. ( 2005). Self-similarity of complex networks. Nature 433, 392-395.

36. Song, C., Havlin, S. and Makse, H.A. ( 2006). Origins of fractality int he growth of complex networks. Nat Phys. 2, 275-281.

37. Tian, X.; Xiao, Z.G. (October 2005). Functional Model of Brainstem-Cortex-Thalamus Circuit. In Proceedings of the 2005 Neural International Conference on Networks and Brain, ICNN\&B '05, Beijing, China, 13-15; Volume 3.

38. Tsuda, I.; Yamaguti, Y.; Kuroda, S.; Fukushima, Y.; Tsukada, M. (2008). A mathematical model for the hippocampus: Towards the understanding of episodic memory and imagination. Prog. Theor. Phys. Suppl., 173, 99-108.

39. Wang N, Butler JP, Ingber DE. (1993). Mechanotransduction across the cell surface and through the cytoskeleton. Science; 260: 1124-1127.

40. Werner, G. (2010). Fractals int he nervous system: conceptual implications for theoretical neuroscience. Front. Physiol. 1:15.

41. West B.J. (2010). Fractal physiology and the fractional calculus: a perspective. Front Physiol 1:12.

42. Zanotti, G., Guerra C. (2003). Is tensegrity a unifying concept of protein folds? FEBS Lett; 534: 7-10. 